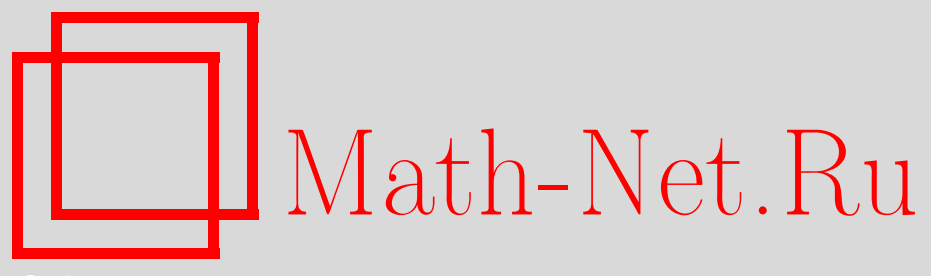

Д. В. Прохоров, С. В. Романова, Локальные экстремальные задачи для ограниченных аналитических функций без нулей, Изв. РАН. Сер. матем., 2006, том 70, выпуск 4, 209-224

DOI: https://doi.org/10.4213/im564

Использование Общероссийского математического портала Math-Net.Ru подразумевает, что вы прочитали и согласны с пользовательским соглашением http://www . mathnet.ru/rus/agreement

Параметры загрузки:

IP: 54.224 .60 .19

26 апреля 2023 г., 15:34:00

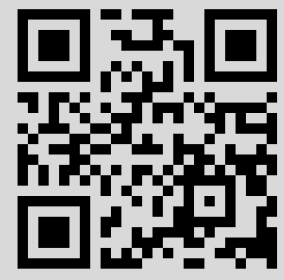


УДК 517.54

\author{
Д. В. Прохоров, С. В. Романова
}

\title{
Локальные экстремальные задачи для ограниченных аналитических функций без нулей
}

\begin{abstract}
В классе $B(t), t>0$, всех аналитических в единичном круге $U$ функций $f(z, t)=e^{-t}+c_{1}(t) z+c_{2}(t) z^{2}+\ldots$, удовлетворяющих в $U$ условию $0<|f(z, t)|<1$, найдены асимптотические оценки коэффициентов при малых и достаточно больших $t>0$. Приведен алгоритм определения тех $t>0$, при которых канонические функции доставляют локальный максимум $\operatorname{Re} c_{n}(t)$ в классе $B(t)$. Описано множество функционалов $L(f)=\sum_{k=0}^{n} \lambda_{k} c_{k}$, для которых канонические функции доставляют максимум $\operatorname{Re} L(f)$ в классе $B(t)$ при малых и больших значениях $t$. Доказательства основаны на применении методов оптимизации для решений управляемой системы дифференциальных уравнений.
\end{abstract}

Библиография: 17 наименований.

\section{$\S 1$. Введение}

Обозначим через $B$ класс аналитических в единичном круге $U=\{z:|z|<1\}$ функций

$$
f(z)=\sum_{n=0}^{\infty} c_{n} z^{n}, \quad 0<|f(z)|<1, \quad z \in U .
$$

В последние десятилетия изучение функций класса $B$ привлекает внимание математиков. Классические и новые методы привели к решению ряда экстремальных задач [1]-[11] в классе $B$ и его подклассах. Однако наиболее притягательная гипотеза Кжижа [12] о том, что справедливо равенство

$$
\max _{f \in B}\left|c_{n}\right|=\frac{2}{e}, \quad n \geqslant 1,
$$

причем максимум достигается для вращений функции $f(z)=F\left(z^{n}\right)$, где

$$
F(z)=\exp \left\{\frac{z-1}{z+1}\right\}=\frac{1}{e}+\frac{2}{e} z+\ldots, \quad z \in U,
$$

доказана только для $n \leqslant 5$.

На линейном пространстве аналитических в единичном круге $U$ функций с разложением (1) рассмотрим линейный функционал

$$
L(f)=\sum_{k=0}^{n} \lambda_{k} c_{k}, \quad \lambda_{n}=1
$$

Работа выполнена при поддержке РФФИ (грант № 04-01-00083), программы "Университеты России" (грант № УР 04.01.374).

(C) Д. В. Прохоров, С. В. Романова, 2006 
и экстремальную задачу

$$
\operatorname{Re} L(f) \rightarrow \max , \quad f \in B .
$$

Поскольку класс $B$ инвариантен относительно вращений $f(z) \rightarrow e^{i \alpha} f\left(e^{i \beta} z\right)$, $(\alpha, \beta) \in \mathbb{R}^{2}$, сохраняющих модули коэффициентов разложения (1), то экстремальную задачу (4) достаточно рассматривать на сужении класса $B$, состоящем из функций $f \in B$ с неотрицательными коэффициентами $c_{0}$ и $c_{1}$. Заметим, что проблема Кжижа [12] заключается в решении задачи (4) для функционала $L$, задаваемого формулой (3) с параметрами $\lambda_{0}=\lambda_{1}=\cdots=\lambda_{n-1}=0, \quad \lambda_{n}=1$.

Считая коэффициент $c_{0}$ неотрицательным и пользуясь условием $0<c_{0}<1$, положим $c_{0}=e^{-t}, 0<t<\infty$. Такая нормировка производит расслоение класса функций $f \in B$ с положительным коэффициентом $c_{0}$ на семейство классов $B(t)=\left\{f \in B: c_{0}=e^{-t}\right\}, \quad t>0$. Класс $B(t), t>0$, является компактным в топологии равномерной сходимости на компактных подмножествах круга $U$, поэтому всякий непрерывный функционал достигает на нем своего максимума.

В настоящей статье найдены асимптотические оценки коэффициентов функций класса $B(t)$ при малых и достаточно больших $t>0$ и дается алгоритм определения тех $t>0$, при которых канонические функции доставляют локальный максимум $\operatorname{Re} c_{n}$ в классе $B(t)$. Описано множество функционалов $(3)$, для которых канонические функции являются экстремальными функциями задачи (4) в классе $B(t)$ при малых и больших значениях $t>0$.

\section{§ 2. Основные сведения о параметрическом методе и оптимизации}

Очевидно, что существует простое соотношение между классами $B(t)$ и классом Каратеодори $P$, состоящем из аналитических в $U$ функций $p, p(z)=$ $1+p_{1} z+\ldots, \operatorname{Re} p(z)>0, z \in U$. Действительно, $f \in B(t), t>0$, в том и только в том случае, когда $p=(-1 / t) \log f \in P$. Класс $P$ изучен довольно подробно, его структура определяется интегральным представлением РиссаГерглотца (см., например, [13, с. 61]).

Всякий функционал на классе $B(t)$, аналитически зависящий от коэффициентов $c_{1}, \ldots, c_{n}$ разложения (1), взаимно однозначно соответствует функционалу на классе $P$, аналитически зависящему от тейлоровских коэффициентов $p_{1}, \ldots, p_{n}$ функции $p \in P$. Как известно (см., например, [13, с. 124]), функции, доставляющие экстремум таким функционалам в классе $P$, содержатся в семействе функций

$$
\begin{gathered}
p(z)=\sum_{k=1}^{m} \nu_{k} \frac{e^{i u_{k}}+z}{e^{i u_{k}}-z} \\
\nu_{k} \geqslant 0, \quad u_{k} \in(-\pi, \pi], \quad k=1, \ldots, m, \quad \sum_{k=1}^{m} \nu_{k}=1, \quad m \leqslant n+1 .
\end{gathered}
$$

Несмотря на простую связь с $P$, класс $B$ оказался вовсе не простым для изучения, а многие коэффициентные оценки в нем остаются неизвестными. Наиболее активное исследование классов $B$ и $B(t)$ началось после появления статьи [6], в которой были определены задачи и намечены некоторые пути их решения. В частности, была найдена оценка $\left|c_{2}\right|$ в классе $B(t)$ при всех $t>0$. Оценка $\left|c_{3}\right|$ в зависимости от $t>0$ была позднее получена в работах [9], [10]. 
Помимо прочего, в [6] было показано, что класс $B(t)$ имеет своеобразное параметрическое представление. Именно, для всякой функции $f$ из некоторого всюду плотного подмножества класса $B\left(t_{0}\right), t_{0}>0$, найдется измеримая функция $u=u(t), t \geqslant 0$, такая, что $f(z)=w\left(z, t_{0}\right)$, где $w=w(z, t)$ является интегралом обыкновенного дифференциального уравнения типа Левнера

$$
\frac{d w}{d t}=-w \frac{e^{i u}+z}{e^{i u}-z}, \quad w(z, 0)=1, \quad 0 \leqslant t \leqslant t_{0}, \quad z \in U .
$$

Обратно, решение $w=w\left(z, t_{0}\right)$ дифференциального уравнения (6) с произвольной измеримой функцией $u=u(t)$ принадлежит классу $B\left(t_{0}\right)$. Функции $f=\exp \left\{-t_{0} p\right\}$, где $p$ задаются формулой (5), представимы решениями дифференциального уравнения (6). Поэтому максимум функционалов в задаче (4) достигается на решениях уравнения (6).

Параметрическое представление решениями дифференциального уравнения (6) позволяет применять оптимизационные методы для решения экстремальных задач в классах $B(t)$. Изложим оптимизационную схему принципа максимума Понтрягина [14] как необходимого условия максимума в задаче (4).

Пусть

$$
w(z, t)=\sum_{k=0}^{\infty} c_{k}(t) z^{k}, \quad 0 \leqslant t \leqslant t_{0}, \quad z \in U,
$$

является решением дифференциального уравнения (6) с заданной управляющей функцией $u=u(t)$ и $f(z)=w\left(z, t_{0}\right)$ имеет разложение $(1)$.

Подставим разложение (7) в дифференциальное уравнение (6), приравняем коэффициенты при одинаковых степенях $z$ в обеих частях уравнения (6) и получим систему дифференциальных уравнений относительно $a(t)=\left(a_{0}(t), \ldots\right.$ $\left.\ldots, a_{n}(t)\right)=e^{t} c(t)=\left(e^{t} c_{0}(t), \ldots, e^{t} c_{n}(t)\right):$

$$
\begin{gathered}
\frac{d a_{0}}{d t}=0, \quad a_{0}(0)=1, \quad \frac{d a_{j}}{d t}=-2 \sum_{s=1}^{j} e^{i s u} a_{j-s}, \\
a_{j}(0)=0, \quad j=1, \ldots, n .
\end{gathered}
$$

Первое уравнение системы (8) влечет тождественное равенство $a_{0}(t)=1$. Экстремальная задача $(4)$ в классе $B\left(t_{0}\right)$ запишется в виде

$$
\sum_{k=1}^{n}\left(\operatorname{Re} \lambda_{k} \operatorname{Re} a_{k}\left(t_{0}\right)-\operatorname{Im} \lambda_{k} \operatorname{Im} a_{k}\left(t_{0}\right)\right) \rightarrow \max .
$$

Уравнения системы (8) запишем в комплексной векторной форме:

$$
\frac{d a}{d t}=G(t, a, u)
$$

где $G(t, a, u)=\left(0, G_{1}(t, a, u), \ldots, G_{n}(t, a, u)\right)$ - вектор правых частей уравнений системы (8). Обозначим

$$
\begin{array}{ll}
\operatorname{Re} a_{k}=x_{2 k-1}, & \operatorname{Im} a_{k}=x_{2 k}, \\
\operatorname{Re} G_{k}(t, a, u)=g_{2 k-1}(t, a, u), & \operatorname{Im} G_{k}(t, a, u)=g_{2 k}(t, a, u),
\end{array}
$$


где $k=1, \ldots, n$, и положим

$$
x=\left(x_{1}, \ldots, x_{2 n}\right), \quad g(t, x, u)=\left(g_{1}(t, x, u), \ldots, g_{2 n}(t, x, u)\right) .
$$

Уравнения системы (8) можно записать в действительной векторной форме:

$$
\frac{d x}{d t}=g(t, x, u)
$$

Функция Гамильтона задачи (9) имеет вид [14, с. 79-80]

$$
\begin{aligned}
H(t, x, \varphi, u)=- & \varphi_{0}\left(g_{2 n-1}+\operatorname{Re} \lambda_{n-1} g_{2 n-3}\right. \\
& \left.\quad-\operatorname{Im} \lambda_{n-1} g_{2 n-2}+\cdots+\operatorname{Re} \lambda_{1} g_{1}-\operatorname{Im} \lambda_{1} g_{2}\right)+\sum_{k=1}^{2 n} \varphi_{k} g_{k} .
\end{aligned}
$$

Обозначим

$$
\begin{aligned}
& \psi_{n}=\varphi_{2 n-1}-\varphi_{0}-i \varphi_{2 n}, \\
& \psi_{n-1}=\varphi_{2 n-3}-\operatorname{Re} \lambda_{n-1} \varphi_{0}-i\left(\varphi_{2 n-2}+\operatorname{Im} \lambda_{n-1} \varphi_{0}\right), \\
& \psi_{1}=\varphi_{1}-\operatorname{Re} \lambda_{1} \varphi_{0}-i\left(\varphi_{2}+\operatorname{Im} \lambda_{1} \varphi_{0}\right) .
\end{aligned}
$$

Таким образом, функцию Гамильтона задачи (9) можно записать в комплексной форме:

$$
H(t, a, \Psi, u)=-2 \operatorname{Re} \sum_{j=1}^{n} \sum_{s=1}^{j} e^{i s u} a_{j-s} \Psi_{j},
$$

где комплекснозначные координаты вектора $\Psi=\left(\Psi_{1}, \ldots, \Psi_{n}\right)$ удовлетворяют сопряженной системе дифференциальных уравнений

$$
\begin{gathered}
\frac{d \Psi_{j}}{d t}=2 \sum_{s=1}^{n-j} e^{i s u} \Psi_{s+j}, \quad j=1, \ldots, n-1, \\
\frac{d \Psi_{n}}{d t}=0
\end{gathered}
$$

с условиями трансверсальности на правом конце отрезка $\left[0, t_{0}\right]$ :

$$
\Psi_{j}\left(t_{0}\right)=\lambda_{j}, \quad j=1, \ldots, n .
$$

Последнее уравнение системы (11) вместе с условиями трансверсальности (12) влечет тождественные равенства $\Psi_{n}=\lambda_{n}=1$.

Оптимальное управление $u=u(t)$ доставляет во всех точках непрерывности на отрезке $\left[0, t_{0}\right]$ максимум функции Гамильтона (10) вдоль оптимальной траектории $(a, \Psi)$ систем (8) и $(11)$.

Функция Гамильтона (10) как функция переменного $u$ может иметь несколько точек максимума на $(-\pi, \pi]$, что соответствует так называемому скользящему режиму. Р. В. Гамкрелидзе [15] предложил в таком случае для выбора оптимального управления перейти к новой фазовой системе, правая часть которой представляет собой выпуклую комбинацию правых частей прежней системы 
со своими управлениями. Таким образом, перейдем от фазовой системы (8) к системе дифференциальных уравнений

$$
\begin{gathered}
\frac{d a_{j}}{d t}=-2 \sum_{k=1}^{m} \nu_{k} \sum_{s=1}^{j} e^{i s u_{k}} a_{j-s}, \quad a_{j}(0)=0, \quad j=1, \ldots, n, \\
a_{0}=1, \quad \nu_{1} \geqslant 0, \ldots, \nu_{m} \geqslant 0, \quad \sum_{k=1}^{m} \nu_{k}=1 .
\end{gathered}
$$

Здесь $m$ равно числу точек максимума функции Гамильтона на $(-\pi, \pi], a_{k}(t)=$ $e^{t} c_{k}(t), \quad k=0, \ldots, n, c_{k}(t)$ определены разложением (7). Скалярное управление $u$ заменяется на векторное управление $u=\left(u_{1}, \ldots, u_{m}\right)$, а коэффициенты $\nu_{1}, \ldots, \nu_{m}$ в общем случае зависят от $t$ и являются управлениями.

Покажем, что в задаче (9), (13) можно положить $m \leqslant n$ и выбор оптимальных управлений сосредоточить лишь на постоянных управляющих функциях.

ЛЕмма 1. В задаче оптимального управления (9), (13) существуют постоянные неотрицательные коэффициенты $\nu_{1}, \ldots, \nu_{m}, \sum_{k=1}^{m} \nu_{k}=1, m \leqslant n$, $u$ постоянные оптимальные управления $u_{1}, \ldots, u_{m} \in(-\pi, \pi]$.

ДокАЗАтЕльство. Задача оптимального управления (9), (13) эквивалентна экстремальной задаче $(4)$ в классе $B\left(t_{0}\right)$. В силу связи класса $B(t), t>0$, с классом $P$ и описанием множества экстремальных функций для аналитических функционалов в классе $P\left[13\right.$, с. 124] экстремальная функция $f^{*} \in B(t)$ задачи (4) существует и содержится во множестве функций

$$
\begin{gathered}
f(z)=\exp \left\{-t \sum_{k=1}^{m} \nu_{k} \frac{e^{i u_{k}}+z}{e^{i u_{k}}-z}\right\}, \quad \nu_{k} \geqslant 0, \quad u_{k} \in(-\pi, \pi], \quad k=1, \ldots, m, \\
\sum_{k=1}^{m} \nu_{k}=1, \quad m \leqslant n+1 .
\end{gathered}
$$

Каждая функция $f$, заданная формулой (14), является при $t=t_{0}$ решением дифференциального уравнения

$$
\frac{d w}{d t}=-w \sum_{k=1}^{m} \nu_{k} \frac{e^{i u_{k}}+z}{e^{i u_{k}}-z}, \quad w(z, 0)=1, \quad 0 \leqslant t \leqslant t_{0}, \quad z \in U,
$$

с постоянными $\nu_{1}, \ldots, \nu_{m}$ и $u_{1}, \ldots, u_{m}$.

Дифференциальное уравнение (15) взаимно однозначно связано с системой (13). Следовательно, некоторый набор постоянных $\nu_{1}, \ldots, \nu_{m}$ и $u_{1}, \ldots, u_{m}$ является оптимальным управляющим вектором в задаче (9), (13).

Осталось показать, что $m \leqslant n$. Предположим, что $m=n+1$. Тогда найдется набор положительных постоянных $\nu_{1}, \ldots, \nu_{n+1}, \quad \sum_{k=1}^{n+1} \nu_{k}=1$, и различных постоянных $u_{1}, \ldots, u_{n+1} \in(-\pi, \pi]$, удовлетворяющих необходимому условию максимума в задаче (9). В частности, для каждого $t \in\left[0, t_{0}\right]$ точки $u_{1}, \ldots, u_{n+1}$ являются точками максимума функции Гамильтона (10). Условие $\Psi_{n}=1$ обеспечивает невырожденность функции Гамильтона, которая представляет собой тригонометрический многочлен точной степени $n$. Поэтому функция Гамильтона может иметь не более $n$ точек максимума на $(-\pi, \pi]$. Полученное противоречие означает, что $m \leqslant n$. Таким образом, лемма доказана. 
Выделим два наиболее характерных примера функций $f \in B(t)$. Первый из них возникает при выборе $m=1$ и $u=\pi$ в уравнении (15), что дает

$$
w(z, t)=F_{t}(z)=\exp \left\{-t \frac{1-z}{1+z}\right\}=\sum_{k=0}^{\infty} A_{k}(t) z^{k} .
$$

При $t=1$ функция $F_{1}(z)$ дается формулой $(2)$.

Другой пример возникает при $m=n, \nu_{1}=\cdots=\nu_{n}=1 / n$ и $u_{1}, \ldots, u_{n}$, являющихся корнями уравнения $u^{n}=-1$ на $(-\pi, \pi]$, что дает $w(z, t)=F_{t}\left(z^{n}\right)$. Роль этого примера особенно значима в связи с предположением об экстремальности $F_{1}\left(z^{n}\right)$ в гипотезе Кжижа.

\section{§ 3. Асимптотические оценки коэффициентов в случае $f(0)=1$}

Поскольку $w(z, 0)=1$ в уравнении $(15)$, то для его решений $w(z, t)$ справедливо предельное соотношение $\lim _{t \rightarrow 0} w(z, t)=1$. Р. Перец [8] доказал, что для всякого $n \geqslant 1$ существует $t_{n}>0$ такое, что для всех $t \in\left[0, t_{n}\right]$

$$
\max _{f \in B(t)}\left|c_{n}\right|=\left|A_{1}(t)\right|
$$

с экстремальной функцией $F_{t}\left(z^{n}\right)$. Это равносильно решению экстремальной задачи (4) в классах $B(t)$ с функционалом (3) при $\lambda_{1}=\cdots=\lambda_{n-1}=0$. Второй автор настоящей статьи показала [16], что из всех функционалов вида (3) только для $L(f)=c_{n}$ справедливо утверждение Переца.

Мы предложим здесь оптимизационный вариант доказательства, что позволит в следующем параграфе найти точные значения $t$, при которых функция $F_{t}\left(z^{n}\right)$ дает локальный максимум $\operatorname{Re} c_{n}$ в классе $B(t)$.

Положим

$$
\begin{gathered}
u_{k}^{0}=\frac{-\pi+2 k \pi}{n}, \quad k=1, \ldots, n, \\
B_{k}=\left(\cos u_{k}^{0}, \sin u_{k}^{0}, \ldots, \cos \left((n-1) u_{k}^{0}\right), \sin \left((n-1) u_{k}^{0}\right)\right), \quad k=1, \ldots, n .
\end{gathered}
$$

Векторы $B_{k}$ имеют размерность $2 n-2$.

Нам потребуется следующее вспомогательное утверждение.

Лемма 2. Векторы $B_{k}-B_{n}, k=1, \ldots, n-1$, линейно независимы.

ДокАЗАТЕЛЬСтво. Предположим, что векторы $B_{1}-B_{n}, \ldots, B_{n-1}-B_{n}$ линейно зависимы. Значит, найдутся числа $\mu_{1}, \ldots, \mu_{n}$, не равные одновременно нулю, такие, что

$$
\sum_{k=1}^{n-1} \mu_{k}\left(B_{k}-B_{n}\right)=0 \text {. }
$$

Положим $\mu_{n}=-\mu_{1}-\cdots-\mu_{n-1}$. Тогда последнее равенство равносильно

$$
\sum_{k=1}^{n} \mu_{k} B_{k}=0, \quad \sum_{k=1}^{n} \mu_{k}=0
$$


или в координатном виде

$$
\sum_{k=1}^{n} \mu_{k} \cos \left(s u_{k}^{0}\right)=\sum_{k=1}^{n} \mu_{k} \sin \left(s u_{k}^{0}\right)=0, \quad s=1, \ldots, n-1 .
$$

Обозначим

$$
\begin{gathered}
W_{k}(z)=\frac{1}{e^{i u_{k}^{0}}-z}=\sum_{j=0}^{\infty} e^{-i(j+1) u_{k}^{0}} z^{j}, \\
W(z)=\sum_{k=1}^{n} \mu_{k} W_{k}(z)=\sum_{j=0}^{\infty} \sum_{k=1}^{n} \mu_{k} e^{-i(j+1) u_{k}^{0}} z^{j} .
\end{gathered}
$$

Отсюда и из равенств (18) следует, что

$$
W(0)=0, \quad \ldots, \quad W^{(n-2)}(0)=0 .
$$

Преобразуя $W(z)$ к рациональному виду, находим, что

$$
W(z)=\frac{Q(z)}{\Pi_{k=1}^{n}\left(e^{i u_{k}^{0}}-z\right)},
$$

причем благодаря второму равенству (17) многочлен $Q(z)$ имеет степень не выше $n-2$. Это противоречит условию (19). Лемма доказана.

Теперь получим качественное описание экстремальной функции в задаче об оценке $\operatorname{Re} c_{n}$ в случае $f(0)$, близких к 1 .

Лемма 3. Функиия, доставляющая максимум $\operatorname{Re} c_{n}$ в классе $B(t)$ для достаточно малых $t>0$, является решением дифференциального уравнения (15) с $m=n$, положительными постоянными $\nu_{1}, \ldots, \nu_{n}$ и различными $u_{1}, \ldots, u_{n} \in$ $(-\pi, \pi]$.

ДокАЗАТЕЛьство. В экстремальной задаче (9) о максимуме $\operatorname{Re} c_{n}$ в классе $B(t)$ предполагается, что $\lambda_{1}=\cdots=\lambda_{n-1}=0$. Фазовой системе (13) соответствует сопряженная система дифференциальных уравнений

$$
\frac{d \Psi_{j}}{d t}=2 \sum_{k=1}^{m} \nu_{k} \sum_{s=1}^{n-j} e^{i s u_{k}} \Psi_{s+j}, \quad j=1, \ldots, n-1,
$$

с условиями трансверсальности (12), из которой следует, что

$$
\Psi_{j}=o(1), \quad j=1, \ldots, n-1, \quad t \rightarrow 0 .
$$

По этой причине согласно (10) имеем

$$
H(0, a(0), \Psi(0), u)=-2 \cos (n u)+o(1),
$$

а значит, все возможные точки $u_{k}$ максимума функции Гамильтона имеют асимптотику

$$
u_{k}=u_{k}^{0}+o(1), \quad k=1, \ldots, n, \quad u_{k} \rightarrow u_{k}^{0} .
$$


Из последнего уравнения системы (13) заключаем, что

$$
\left.\frac{d \operatorname{Re} a_{n}}{d t}\right|_{t=0}=-2 \sum_{k=1}^{m} \nu_{k} \cos \left(n u_{k}\right) \leqslant 2
$$

со знаком равенства для $m=n$ и $u_{1}=u_{1}^{0}, \ldots, u_{n}=u_{n}^{0}$. Принимая $u_{1}, \ldots, u_{n}$ постоянными, продифференцируем по $t$ последнее уравнение системы (13) при $m=n$ и получим

$$
\begin{aligned}
\frac{d^{2} a_{n}}{d t^{2}} & =-2 \sum_{k=1}^{n} \nu_{k} \sum_{s=1}^{n} e^{i s u_{k}} \frac{d a_{n-s}}{d t} \\
& =-2 \sum_{k=1}^{n} \nu_{k} \sum_{s=1}^{n} e^{i s u_{k}}(-2) \sum_{l=1}^{n} \nu_{l} \sum_{\sigma=1}^{n-s} e^{i \sigma u_{k}} a_{n-s-\sigma}
\end{aligned}
$$

Пользуясь начальными условиями в (13) и тем, что $a_{0}=1$, из $(23)$ находим

$$
\begin{aligned}
\left.\frac{d^{2} a_{n}}{d t^{2}}\right|_{t=0} & =4 \sum_{k=1}^{n} \sum_{l=1}^{n} \sum_{s=1}^{n} e^{i s u_{k}} e^{i(n-s) u_{l}} \nu_{k} \nu_{l} \\
& =4 \sum_{k=1}^{n} \sum_{l=1}^{n} \sum_{s=1}^{n} e^{i s u_{k}^{0}} e^{i(n-s) u_{l}^{0}} \nu_{k} \nu_{l}+o(1) \\
& =-4 \sum_{k=1}^{n} \sum_{l=1}^{n} \sum_{s=1}^{n} e^{i s\left(u_{k}^{0}-u_{l}^{0}\right)} \nu_{k} \nu_{l}+o(1)
\end{aligned}
$$

при $u_{k} \rightarrow u_{k}^{0}$. Поскольку

$$
\sum_{s=1}^{n} e^{i s\left(u_{k}^{0}-u_{l}^{0}\right)}=\left\{\begin{array}{lll}
0 & \text { при } & k \neq l \\
n & \text { при } & k=l
\end{array}\right.
$$

то из (24) следует, что

$$
\left.\frac{d^{2} a_{n}}{d t^{2}}\right|_{t=0}=-4 n \sum_{k=1}^{n} \nu_{k}^{2}+o(1)=-4 n\left[\sum_{k=1}^{n-1} \nu_{k}^{2}+\left(1-\sum_{k=1}^{n-1} \nu_{k}\right)^{2}\right]+o(1)
$$

при $u_{k} \rightarrow u_{k}^{0}$.

Абсолютный максимум квадратичной формы

$$
q_{n-1}=-4 n \sum_{k=1}^{n} \nu_{k}^{2}=-4 n\left[\sum_{k=1}^{n-1} \nu_{k}^{2}+\left(1-\sum_{k=1}^{n-1} \nu_{k}\right)^{2}\right]
$$

равный -4 , достигается в точке $\left(\nu_{1}, \ldots, \nu_{n-1}\right)=(1 / n, \ldots, 1 / n)$, которая является внутренней точкой симплекса

$$
S=\left\{\left(\nu_{1}, \ldots, \nu_{n-1}\right): \nu_{1} \geqslant 0, \ldots, \nu_{n-1} \geqslant 0, \sum_{k=1}^{n-1} \nu_{k} \leqslant 1\right\} .
$$

Абсолютный максимум квадратичной формы $q_{n-1}$ на границе $\partial S$ симплекса $S$ равен $-4 n /(n-1)$. 
Из соотношений $(22)$ и $(25)$ следует, что

$$
\operatorname{Re} a_{n}(t)=\operatorname{Re}\left[a_{n}^{\prime}(0) t+\frac{a_{n}^{\prime \prime}(0)}{2} t^{2}+o\left(t^{2}\right)\right] \leqslant 2 t-2 t^{2}+o\left(t^{2}\right)
$$

со знаком равенства для $m=n$ и $u_{1}=u_{1}^{0}, \ldots, u_{n}=u_{n}^{0}$.

Выберем $t>0$ настолько близким к нулю, чтобы модули величин $o(1)$ и $o\left(t^{2}\right) / t^{2}$ в $(25)$ и $(26)$ не превышали $1 /(2(n-1))$. Переход от скользящего режима с $m=n$ в задаче оптимального управления (9), (13) к режиму с меньшим числом $m$ означает, что один из коэффициентов $\nu_{1}, \ldots, \nu_{m}$ равен нулю, а это равносильно переходу к границе $\partial S$ симплекса $S$ множества значений $\nu_{1}, \ldots, \nu_{n-1}$. В таком случае $\operatorname{Re} a_{n}(t) \leqslant 2 t-(2+1 /(n-1)) t^{2}$, и, сравнивая c (26), получаем утверждение леммы.

Лемма 4. Множество $\Sigma$ точек $\xi=\left(\xi_{1}, \ldots, \xi_{n-1}, 1\right)=\Psi(0)$, для которых функиия Гамильтона при $t=0$ имеет $n$ точек максимума на $(-\pi, \pi]$, является гладкой поверхностью вещественной размерности $n-1$ в окрестности точки $\xi^{0}=(0, \ldots, 0,1)$.

ДокАЗАТЕЛЬСтво. Условие наличия $n$ точек максимума $u_{1}, \ldots, u_{n}$ функции Гамильтона (10) при $t=0$ выражается равенствами

$$
\begin{aligned}
H_{u}\left(0, a(0), \xi, u_{1}\right) & =\cdots=H_{u}\left(0, a(0), \xi, u_{n}\right)=0, \\
H\left(0, a(0), \xi, u_{1}\right) & =\cdots=H\left(0, a(0), \xi, u_{n}\right) .
\end{aligned}
$$

Первая серия равенств определяет точки $u_{1}, \ldots, u_{n}$ в зависимости от $\xi$, а вторая серия равенств, преобразованная к виду

$$
\operatorname{Re} \sum_{j=1}^{n} e^{i j u_{1}} \xi_{j}=\cdots=\operatorname{Re} \sum_{j=1}^{n} e^{i j u_{n}} \xi_{j},
$$

описывает множество точек $\xi$.

Пусть $\xi_{j}=\eta_{2 j-1}+i \eta_{2 j}, j=1, \ldots, n-1$. Равенства $(27)$ выполняются в точке $\xi^{0}$ и определяют гладкую неявную функцию $n-1$ переменных вещественных координат вектора $\xi$ от остальных свободных координат этого вектора, если матрица системы уравнений (27), составленная из элементов

$$
\cos \left(j u_{k}\right)-\cos \left(j u_{n}\right), \quad \sin \left(j u_{k}\right)-\sin \left(j u_{n}\right),
$$

$j=1, \ldots, n-1, \quad k=1, \ldots, n-1$, имеет максимальный ранг. Строки матрицы с элементами (28) состоят из векторов $B_{1}-B_{n}, \ldots, B_{n-1}-B_{n}$, которые по лемме 2 линейно независимы. Таким образом, лемма доказана.

Выразим схему асимптотического описания поверхности $\Sigma$. Положим $\eta_{j}=$ $\varepsilon e_{j}+o(\varepsilon), j=1, \ldots, 2 n-2$. Продифференцируем равенства $(27)$ по координатам вектора $\xi$ в точке $\varepsilon=0$ и получим систему линейных уравнений с матрицей, составленной из элементов $(28)$ с заменой $\eta_{j}$ на $e_{j}, j=1, \ldots, 2 n-2$. Максимальность ранга системы позволяет определить $n-1$ вещественных координат вектора $\xi$ как функции остальных координат. Свободные координаты вектора $\xi$ будем обозначать $\eta_{j_{1}}, \ldots, \eta_{j_{n-1}}$. 
Лемма 5. Пусть в задаче оптимального управления (9), (13) $\lambda_{1}=\ldots$ $\cdots=\lambda_{n-1}=0, \lambda_{n}=1, \Psi(0)=\xi^{0}$. Тогда управления $u_{1}=u_{1}^{0}, \ldots, u_{n}=u_{n}^{0}$ удовлетворяют принципу максимума Понтрягина, а вектор $\Psi$ удовлетворяет условиям трансверсальности (12) при $\nu_{1}=\cdots=\nu_{n}=1 / n$.

ДокАЗАТЕЛЬство. Проверка утверждений леммы осуществляется непосредственно. Действительно, правые части систем (13) и (20) обращаются в нуль, поэтому векторы $a(t)$ и $\Psi(t)$ остаются постоянными, как и функция Гамильтона $H(t, a, \Psi, u)=-2 \cos (n u), t \geqslant 0$. Лемма доказана.

Теорема 1. Для всякого $n \geqslant 1$ существует $t_{n}>0$ maкое, что для всех $t \in\left[0, t_{n}\right]$

$$
\max _{f \in B(t)} \operatorname{Re} c_{n}=\left|A_{1}(t)\right|
$$

ДокАЗАТЕЛЬСтво. Пусть $t_{n}>0$. Обозначим $\nu=\left(\nu_{1}, \ldots, \nu_{n-1}\right), \nu_{n}=1-$ $\sum_{k=1}^{n-1} \nu_{k}, \quad \nu^{0}=(1 / n, \ldots, 1 / n), \quad \mu=\left(\mu_{1}, \ldots, \mu_{n-1}\right)=t_{n}\left(\nu-\nu^{0}\right)$ и рассмотрим отображение

$$
G:(\mu, \xi) \rightarrow \Psi\left(t_{n}\right)
$$

где $\mu$ принадлежит окрестности $O_{\mu}$ точки $0=(0, \ldots, 0), \quad \xi$ принадлежит окрестности $O_{\xi}$ точки $\xi^{0}, O_{\mu}, O_{\xi} \subset \Sigma$, а $\Psi(t)$ является решением системы дифференциальных уравнений (20) с $m=n$, в которой точки $u_{1}, \ldots, u_{n}$ удовлетворяют принципу максимума Понтрягина в момент $t=0$ для функции Гамильтона (10) с фазовыми значениями $a$, заданными системой (13). Заметим, что $G\left(0, \xi^{0}\right)=\Psi\left(t_{n}\right)=(0, \ldots, 0,1)$ и точка $\left(0, \xi^{0}\right)$ согласно (15) соответствует функции $F_{t_{n}}\left(z^{n}\right)$.

Найдем частную производную $G_{\mu}$ :

$$
\frac{\partial G}{\partial \mu}=\left(\frac{\partial G}{\partial \mu_{1}}, \ldots, \frac{\partial G}{\partial \mu_{n-1}}\right)
$$

Поскольку

$$
\Psi_{j}\left(t_{n}\right)=2 \int_{0}^{t_{n}}\left[\sum_{k=1}^{n-1} \nu_{k} \sum_{s=1}^{n-j} e^{i s u_{k}} \Psi_{s+j}+\left(1-\sum_{k=1}^{n-1} \nu_{k}\right) \sum_{s=1}^{n-j} e^{i s u_{n}} \Psi_{s+j}\right] d t
$$

Tо

$$
\begin{aligned}
\frac{\partial \Psi_{j}}{\partial \mu_{k}} & =\frac{2}{t_{n}} \int_{0}^{t_{n}}\left[\sum_{s=1}^{n-j} e^{i s u_{k}} \Psi_{s+j}-\sum_{s=1}^{n-j} e^{i s u_{n}} \Psi_{s+j}\right] d t \\
& =-2\left(e^{-i j u_{k}}-e^{-i j u_{n}}\right)+o(1), \quad j=1, \ldots, n-1, \quad k=1, \ldots, n-1 .
\end{aligned}
$$

Таким образом, строки матрицы $G_{\mu}$ с точностью до о $(1)$ и знаков нечетных координат состоят из векторов $2\left(B_{k}-B_{n}\right), k=1, \ldots, n-1$, которые линейно независимы по лемме 2.

Перейдем к вычислению частной производной $G_{\xi}$, которая является матрицей со строками, содержащими частные производные координат вектора $\Psi\left(t_{n}\right)$ по свободным координатам вектора $\xi$. Поскольку $\Psi(0)=\xi$, то при $t=0$ прямоугольная матрица $G_{\xi}$ размерностью $(n-1) \times(2 n-2)$ содержит единичную 
матрицу размерностью $(n-1) \times(n-1)$ с номерами столбцов $j_{1}, \ldots, j_{n-1}$. Следовательно, строки матрицы $G_{\xi}$ линейно независимы при $t=0$, а значит, и при достаточно малых $t>0$.

Наконец, условие скользящего режима требует выполнения равенств

$$
H\left(0, a(0), \xi, u_{k}\right)=H\left(0, a(0), \xi, u_{n}\right), \quad k=1, \ldots, n-1,
$$

или с использованием формулы (10)

$$
\operatorname{Re} \sum_{j=1}^{n-1}\left(e^{i j u_{k}}-e^{i j u_{n}}\right) \xi_{j}=0, \quad k=1, \ldots, n-1 .
$$

Продифференцируем это равенство по свободным координатам $\eta_{j_{1}}, \ldots, \eta_{j_{n-1}}$ вектора $\xi$, в результате чего получим равенства, означающие, что все векторы $B_{1}-B_{n}, \ldots, B_{n-1}-B_{n}$ ортогональны каждой из строк матрицы $G_{\xi}$ при $t=0$, откуда следует, что строки $B_{1}-B_{n}, \ldots, B_{n-1}-B_{n}$ и строки матрицы $G_{\xi}$ при $t=0$ вместе образуют обратимую квадратную матрицу размерностью $(2 n-2) \times(2 n-2)$.

Полная производная $G^{\prime}$ отображения $G$ является квадратной матрицей, которая образуется соединением прямоугольных матриц $G_{\mu}$ и $G_{\xi}$, и эта квадратная матрица обратима при достаточно малых $t>0$. По теореме об обратной функции отображение $G$ обратимо и осуществляет взаимно однозначное отображение окрестности $O_{\mu} \times O_{\xi}$ точки $\left(0, \xi^{0}\right)$ на окрестность $O_{\Psi}$ точки $G\left(0, \xi^{0}\right)=(0, \ldots, 0,1)$. Как следует из доказательства теоремы об обратной функции, основанного на принципе Банаха о неподвижной точке сжимающего отображения (см., например, [17, с. 231-234]), радиус окрестности $O_{\xi}$ не меньше, чем величина

$$
\frac{1}{4 \sup _{t \in\left(0, t_{n}\right)}\left\|G^{\prime}\left(0, \xi_{0}\right)\right\|},
$$

которая не уменьшается с уменьшением $t_{n}$, а окрестность $O_{\mu} \times O_{\xi}$ определяется неравенством

$$
\sup _{t \in\left(0, t_{n}\right)}\left\|\left[G^{\prime}\left(0, \xi^{0}\right)\right]^{-1} G^{\prime}(\mu, \xi)-E\right\| \leqslant \frac{1}{2},
$$

где $E$ - единичная матрица.

Уменьшая $t_{n}$, добьемся, чтобы согласно (21)

$$
\Psi(t) \in O_{\xi}, \quad 0 \leqslant t \leqslant t_{n}, \quad G\left(O_{\mu}, O_{\xi}\right) \subset O_{\Psi}
$$

и окрестность $O_{\mu}$ была такова, чтобы соответствующая ей окрестность точки $\nu^{0}$ в пространстве векторов $\nu$ содержала симплекс $S$. При этих условиях точке $\Psi\left(t_{n}\right)=(0, \ldots, 0,1)$, отвечающей условиям трансверсальности $(12)$, соответствует единственная точка $\left(0, \xi^{0}\right)$, описанная в лемме 5 . Значит, в классе $B\left(t_{n}\right)$ одна только функция $F_{t_{n}}\left(z^{n}\right)$ удовлетворяет необходимым условиям оптимальности задачи (9), (13). Таким образом, теорема доказана.

СлЕДСТВИЕ 1. Пусть векторы $\lambda=\left(\lambda_{1}, \ldots, \lambda_{n-1}, 1\right)$ из окрестности точки $(0, \ldots, 0,1)$ задают функииональ $L$ из $(3)$. Тогда разным векторам $\lambda$ соответствуют различные экстремальные функции задачи (4) в классе $B(t)$ при достаточно мальх $t>0$. 
ДокАЗАТЕЛЬство. Вектор $\lambda$ определяет условия трансверсальности (12), которым отображение $G$ устанавливает взаимно однозначное локальное соответствие с вектором $(\nu, \xi), \xi \in \Sigma$, однозначно задающим экстремальные функции задачи (4) в классе $B(t)$. Следствие доказано.

\section{§4. Локальный максимум коэффициентов в случае $f(0)=1$}

Определим те $t>0$, для которых функция $F_{t}\left(z^{n}\right)$ доставляет локальный максимум $\operatorname{Re} c_{n}$ в классе $B(t)$. Воспользуемся аналитическими средствами, согласно которым по лемме 1 локальный максимум $\operatorname{Re} c_{n}$ следует искать на множестве функций (14) с $m=n$, где вектор $\nu$ принимает значения из окрестности $O_{\nu}$ вектора $\nu^{0}$, а вектор $u=\left(u_{1}, \ldots, u_{n}\right)$ - из окрестности $O_{u}$ вектора $u^{0}=\left(u_{1}^{0}, \ldots, u_{n}^{0}\right)$.

Рассмотрим функционал $I=\operatorname{Re} c_{n}$ в классе $B(t)$ и вычислим элементы квадратной матрицы $I^{\prime \prime}$, которая представляет собой производную второго порядка функционала $I$ по $(\nu, u)$ в точке $\left(\nu^{0}, u^{0}\right)$. Матрица $I^{\prime \prime}$ состоит из четырех блоков

$$
\frac{\partial^{2} I}{\partial \nu^{2}}, \quad \frac{\partial^{2} I}{\partial \nu \partial u}, \quad \frac{\partial^{2} I}{\partial u \partial \nu}=\left(\frac{\partial^{2} I}{\partial \nu \partial u}\right)^{T}, \quad \frac{\partial^{2} I}{\partial u^{2}} .
$$

Пусть функция $f(z)=e^{-t}+\sum_{k=1}^{\infty} c_{k}(\nu, u) z^{k}$ задается формулой (14) с $m=n$. Дифференцируя $f$ по $\nu$, получим

$$
\frac{\partial f}{\partial \nu_{k}}=\exp \left\{-t \sum_{k=1}^{n} \nu_{k} \frac{1+e^{-i u_{k}} z}{1-e^{-i u_{k}} z}\right\}(-t)\left[\frac{1+e^{-i u_{k}} z}{1-e^{-i u_{k}} z}-\frac{1+e^{-i u_{n}} z}{1-e^{-i u_{n} z}}\right]
$$

$k=1, \ldots, n-1$. Еще раз дифференцируя (29) по $\nu$, получим

$$
\begin{aligned}
\frac{\partial^{2} f}{\partial \nu_{k} \partial \nu_{j}}=\exp \left\{-t \sum_{k=1}^{n} \nu_{k} \frac{1+e^{-i u_{k}} z}{1-e^{-i u_{k}} z}\right\} t^{2} \\
\times\left[\frac{1+e^{-i u_{k}} z}{1-e^{-i u_{k}} z}-\frac{1+e^{-i u_{n}} z}{1-e^{-i u_{n} z}}\right]\left[\frac{1+e^{-i u_{j}} z}{1-e^{-i u_{j} z}}-\frac{1+e^{-i u_{n}} z}{1-e^{-i u_{n}} z}\right], \\
k, j=1, \ldots, n-1 .
\end{aligned}
$$

Отсюда найдем

$$
\begin{aligned}
\frac{\partial^{2} I\left(\nu^{0}, u^{0}\right)}{\partial \nu_{k} \partial \nu_{j}} & =e^{-t} 4 t^{2} \operatorname{Re} \sum_{s=1}^{n-1}\left(e^{-i s u_{k}^{0}}-e^{-i s u_{n}^{0}}\right)\left(e^{-i(n-s) u_{j}^{0}}-e^{-i(n-s) u_{n}^{0}}\right) \\
& = \begin{cases}-e^{-t} 4 n t^{2}, & k \neq j, \\
-e^{-t} 8 n t^{2}, & k=j .\end{cases}
\end{aligned}
$$

Дифференцируя (29) по $u$, получаем

$$
\begin{aligned}
& \frac{\partial^{2} f}{\partial \nu_{k} \partial u_{j}}= \exp \left\{-t \sum_{k=1}^{n} \nu_{k} \frac{1+e^{-i u_{k}} z}{1-e^{-i u_{k} z}}\right\} t^{2}\left[\frac{1+e^{-i u_{k}} z}{1-e^{-i u_{k} z}}-\frac{1+e^{-i u_{n}} z}{1-e^{-i u_{n} z}}\right] \\
& \times \nu_{j} \sum_{l=1}^{\infty} 2 l(-i) e^{-i l u_{j}} z^{l}, \\
& k=1, \ldots, n-1, \quad j=1, \ldots, n-1, \quad k \neq j .
\end{aligned}
$$


При $j=k$ или $j=n$ в этих формулах появятся дополнительные слагаемые.

Окончательно отсюда найдем

$$
\begin{aligned}
\frac{\partial^{2} I\left(\nu^{0}, u^{0}\right)}{\partial \nu_{k} \partial u_{j}} & =\frac{e^{-t} 4 t^{2}}{n} \operatorname{Re}(-i) \sum_{s=1}^{n-1}\left(e^{-i s u_{k}^{0}}-e^{-i s u_{n}^{0}}\right)(n-s) e^{-i(n-s) u_{j}^{0}} \\
& = \begin{cases}e^{-t} 2 t^{2}\left(\operatorname{ctg} \frac{u_{k}^{0}-u_{j}^{0}}{2}-\operatorname{ctg} \frac{u_{n}^{0}-u_{j}^{0}}{2}\right), & j \neq k, \\
e^{-t} 2 t^{2} \operatorname{ctg} \frac{u_{k}^{0}-u_{n}^{0}}{2}, & j=k, n,\end{cases}
\end{aligned}
$$

$k=1, \ldots, n-1, j=1, \ldots, n$. Аналогично, дифференцируя $f$ по $u$, вычислим

$$
\frac{\partial f}{\partial u_{k}}=\exp \left\{-t \sum_{k=1}^{n} \nu_{k} \frac{1+e^{-i u_{k}} z}{1-e^{-i u_{k}} z}\right\}(-t) \nu_{k} \sum_{l=1}^{\infty} 2 l(-i) e^{-i l u_{k}} z^{l}, \quad k=1, \ldots, n .
$$

Еще раз дифференцируя эти равенства по $u$, получим

$$
\begin{aligned}
\frac{\partial^{2} f}{\partial u_{k} \partial u_{j}}= & \exp \left\{-t \sum_{k=1}^{n} \nu_{k} \frac{1+e^{-i u_{k}} z}{1-e^{-i u_{k}} z}\right\} t^{2} \nu_{k} \nu_{j} \\
& \times \sum_{l=1}^{\infty} 2 l(-i) e^{-i l u_{k}} z^{l} \sum_{s=1}^{\infty} 2 s(-i) e^{-i s u_{j}} z^{s}, \quad k, j=1, \ldots, n, \quad k \neq j .
\end{aligned}
$$

Отсюда найдем

$$
\begin{aligned}
\frac{\partial^{2} I\left(\nu^{0}, u^{0}\right)}{\partial u_{k} \partial u_{j}} & =\frac{-e^{-t} 4 t^{2}}{n^{2}} \operatorname{Re} \sum_{s=1}^{n-1} s(n-s) e^{-i s\left(u_{k}^{0}-u_{j}^{0}\right)} \\
& =\frac{-e^{-t} 2 t^{2}}{n \sin ^{2}\left(\frac{u_{k}^{0}-u_{j}^{0}}{2}\right)}, \quad k, j=1, \ldots, n, \quad k \neq j .
\end{aligned}
$$

Наконец,

$$
\begin{gathered}
\frac{\partial^{2} f}{\partial u_{k}^{2}}=\exp \left\{-t \sum_{k=1}^{n} \nu_{k} \frac{1+e^{-i u_{k}} z}{1-e^{-i u_{k}} z}\right\}(-t) \nu_{k}\left[(-t) \nu_{k}\left(\sum_{l=1}^{\infty} 2 l(-i) e^{-i l u_{k}} z^{l}\right)^{2}\right. \\
\left.-\sum_{l=1}^{\infty} 2 l^{2} e^{-i l u_{k}} z^{l}\right], \quad k=1, \ldots, n .
\end{gathered}
$$

Отсюда следует, что

$$
\begin{aligned}
\frac{\partial^{2} I\left(\nu^{0}, u^{0}\right)}{\partial u_{k}^{2}} & =\frac{-e^{-t} t}{n}\left[\frac{-4 t}{n} \sum_{s=1}^{n-1} s(n-s)+2 n^{2}\right] \\
& =\frac{e^{-t} 2 t}{3 n}\left(\left(n^{2}-1\right) t-3 n^{2}\right), \quad k=1, \ldots, n .
\end{aligned}
$$

Поскольку $I^{\prime}\left(\nu^{0}, u^{0}\right)=0$, то классическое достаточное условие максимума дважды дифференцируемого функционала $I$ приводит к следующей теореме.

Теорема 2. Пусть $t_{n}>0$ обладает тем свойством, что матрица $I^{\prime \prime}$, состоящая из блоков (30)-(33), отрицательно определена при всех $t \in\left(0, t_{n}\right)$. Тогда функция $F_{t}\left(z^{n}\right)$ доставляет локальный максимум $\operatorname{Re} c_{n}$ в классе $B(t)$ для всех $t \in\left(0, t_{n}\right)$. 


\section{§ 5. Асимптотический и локальный максимумы коэффициентов в случае $f(0)=0$}

Покажем, что функция $F_{t}$ доставляет максимум $\operatorname{Re} c_{n}$ в классе $B(t)$ для достаточно больших $t>0$.

ТЕОрема 3. Для всякого $n \geqslant 1$ существует $T_{n}>0$ mакое, что для всех $t>T_{n}$

$$
\max _{f \in B(t)} \operatorname{Re} c_{n}=\left|A_{n}(t)\right|
$$

ДокАЗАТЕЛЬство. Сначала сравним фазовую и сопряженную системы (8) и (11) или их обобщения (13) и (20) в скользящем режиме с условиями трансверсальности (12) при поиске максимума $\operatorname{Re} c_{n}$ в классе $B\left(t_{0}\right)$. Сравнивая эти системы, обнаруживаем симметрию систем (13) и (20) с обратной нумерацией координат и умножением на -1 . Одновременно начальные условия в (8) или (11) заменяются на аналогичные условия на правом конце отрезка $\left[0, t_{0}\right]$ в (13) или (20), что соответствует изменению направления отсчета параметра $t$ и смене знака производных. Таким образом,

$$
\xi_{k}=\Psi_{k}(0)=a_{n-k}\left(t_{0}\right)=e^{t_{0}} c_{n-k}, \quad k=1, \ldots, n-1 .
$$

Пусть при каждом $t>0$ функция $f(z, t)=e^{-t}+\sum_{k=1}^{\infty} c_{k}(t) z^{k}$ доставляет максимум $\operatorname{Re} c_{n}$ в классе $B(t)$,

$$
f(z, t)=\exp \{-t p(z, t)\}
$$

где $p(z, t) \in P$ при каждом $t>0$. После $n$-кратного дифференцирования равенства (35) обнаруживаем, что

$$
f^{(n)}(0, t)=e^{-t}\left((-t)^{n}\left(p^{\prime}(0, t)\right)^{n}+o\left(t^{n}\right)\right) \quad \text { при } \quad t \rightarrow \infty .
$$

Следовательно,

$$
c_{n}(t)=\frac{e^{-t}}{n !}\left((-t)^{n}\left(p^{\prime}(0, t)\right)^{n}+o\left(t^{n}\right)\right) .
$$

Поскольку $\left|p^{\prime}(0, t)\right| \leqslant 2$, то $-p^{\prime}(0, t)=2 e^{i \beta}+o(1)$ и

$$
\operatorname{Re} c_{n}(t)=\frac{e^{-t} 2^{n} \cos (n \beta)}{n !}\left(t^{n}+o\left(t^{n}\right)\right) .
$$

Одновременно из (35) заключаем, что

$$
c_{k}(t)=\frac{e^{-t} 2^{k} e^{i k \beta}}{k !}\left(t^{k}+o\left(t^{k}\right)\right), \quad k=1, \ldots, n-1 .
$$

Значение функции Гамильтона (10) в начальный момент принимает вид

$$
\begin{aligned}
H(0, a(0), \xi, u) & =-2 \operatorname{Re}\left[e^{i u}\left(\frac{2^{n-1} e^{i(n-1) \beta}}{(n-1) !}\right)\left(t^{n-1}+o\left(t^{n-1}\right)\right)\right] \\
& =-\frac{2^{n} \cos (u+(n-1) \beta)}{(n-1) !}\left(t^{n-1}+o\left(t^{n-1}\right)\right) .
\end{aligned}
$$


При достаточно больших $t$ функция Гамильтона (10) в начальный момент как функция переменного $u$ имеет ровно одну точку максимума на $(-\pi, \pi]$, а значит, согласно (15) экстремальная функция $f(z, t)$ имеет представление $(35)$ с функцией $p(z, t)$, задаваемой формулой $(5)$ с $m=1$, что соответствует вращениям функции $F_{t}$. Теорема доказана.

СлЕДСТВИЕ 2. Пустъ векторы $\lambda=\left(\lambda_{1}, \ldots, \lambda_{n-1}, 1\right)$ из окрестности точки $(0, \ldots, 0,1)$ задают функииональ $L$ из (3). Тогда в задаче (4) им соответствует экстремальная функиия $F_{t}$ в классе $B(t)$ при достаточно больших $t>0$.

ДоказАТЕЛЬСтво. При замене функционала $c_{n}$ на близкие функционалы $L$ при достаточно больших $t$ характер функции Гамильтона в начальный момент сохраняется: она по-прежнему имеет ровно одну точку максимума по переменной $u$ на $(-\pi, \pi]$, что соответствует вращениям функции $F_{t}$ как экстремальным функциям задачи (4) в классе $B(t)$. Следствие доказано.

Следующая теорема позволит определить те $t$, для которых функция $F_{t}$ доставляет локальный максимум $\operatorname{Re} c_{n}$ в классе $B(t)$.

ТЕОРЕма 4. Пусть для $t>0$ тригонометрический многочлен

$$
Q_{n}(u)=-2 \sum_{j=1}^{n} A_{n-j}(t) \cos (j u)
$$

имеет ровно одну точку максимума $u_{0}$ на $(-\pi, \pi]$ u $Q_{n}^{\prime \prime}\left(u_{0}\right)<0$. Тогда функиия $F_{t}$ доставляет локальный максимум $\operatorname{Re} c_{n}$ в классе $B(t)$.

ДоКАЗАТЕЛЬСтво. Воспользуемся соотношениями (34) и, подставляя $\xi_{j}=$ $\Psi_{j}(0)=A_{n-j}(t), \quad j=1, \ldots, n-1$, в функцию Гамильтона $(10)$ в начальный момент $t=0$, заметим, что она имеет ровно одну точку максимума на $(-\pi, \pi]$, и это свойство сохраняется при переходе от коэффициентов $A_{n-j}(t)$ к близким значениям. Значит, в окрестности функции $F_{t}$ экстремальными функциями задачи (4) в классе $B(t)$ могут быть лишь те, которые имеют согласно (15) представление (35) с функцией $p(z, t)$, задаваемой формулой (5) при $m=1$, что соответствует вращениям функции $F_{t}$. Теорема доказана.

\section{Список литературы}

1. Прохоров Д.В., "Коэффициенты голоморфных функций”, Комплексный анализ u теория представлений, Итоги науки и техники. Сер. соврем. матем. и ее прилож. Тематические обзоры, 71, ВИНИТИ, М., 2000.

2. Brown J.E., "On a coefficient problem for nonvanishing $H^{p}$ functions", Compl. Var., 4:3 (1985), 253-265.

3. Tan Delin, "Coefficient estimates for bounded nonvanishing functions", Chinese Ann. Math. A, 4:1 (1983), 97-104.

4. Ermers R., Coefficient estimates for bounded nonvanishing functions, P.H.D. Thesis for grade of Doctor in Mathematics and Information Science, Katholieke Universiteit, Nijmegen, 1990.

5. Horowitz C., "Coefficients of nonvanishing functions in $H^{\infty}$ ", Israel J. Math., 30:3 (1978), 285-291.

6. Hummel J. A., Scheinberg S., Zalcman L., "A coefficient problem for bounded nonvanishing functions", J. Anal. Math., 31 (1977), 169-190. 
7. Lewandowski Z., Szynal J., "On the Krzyz conjecture and related problems", XVIth Rolf Nevanlinna Colloquium, eds. I. Laine, O. Martio, Walter de Gruyter, Berlin, 1996, 257-268.

8. Peretz R., "Applications of subordination theory to the class of bounded nonvanising functions", Compl. Var. Theory Appl., 17:3-4 (1992), 213-222.

9. Prokhorov D.V., Szynal J., "Coefficient estimates for bounded nonvanishing functions", Bull. Acad. Pol. Sci. Ser. Sci. Math., 29:5-6 (1981), 223-230.

10. Prokhorov D. V., Szynal J., "Inverse coefficients for $(\alpha, \beta)$-convex functions", Ann. Univ. M. Curie-Sklodowska. Sect. A., 35 (1981), 125-143.

11. Szapiel W., "A new approach to the Krzyz conjecture", Ann. Univ. M. CurieSklodowska. Sect. A., 48 (1994), 169-192.

12. Krzyz J., "Coefficient problem for bounded nonvanishing functions", Ann. Polon. Mathem., 20 (1967-1968), 314.

13. Александров И. А., Параметрические продолжения в теории однолистных функиий, Наука, М., 1976.

14. Понтрягин Л.С., Болтянский В.Г., Гамкрелидзе Р. В., Мищенко Е.Ф., Математическая теория оптимальных процессов, 3-е изд., Наука, М., 1976.

15. Гамкрелидзе Р. В., Основы теории оптимального управления, Изд-во Тбилисского университета, Тбилиси, 1977.

16. Романова С. В., “Асимптотические оценки линейных функционалов для ограниченных функций, не принимающих нулевого значения", Изв. вузов. Математика, 2002, № 11, 83-85.

17. Рудин У., Основы математического анализа, Мир, М., 1976.

Д. В. Прохоров (D. V. Рrokhorov)

Саратовский государственный

Поступило в редакцию

университет им. Н.Г. Чернышевского

10.11 .2003

E-mail: ProkhorovDV@info.sgu.ru

C. B. Pomahoba (S. V. Romanova)

Саратовский государственный

университет им. Н. Г. Чернышевского

E-mail: RomanovaSV@info.sgu.ru 